\title{
Integrating Human Activities, Archeology, and the Paleo-Critical Zone Paradigm
}

\author{
Joseph V. Ferraro ${ }^{1 *}$, Julie A. Hoggarth ${ }^{1}$, Davide Zori ${ }^{2}$, Katie M. Binetti ${ }^{1}$ and \\ Gary Stinchcomb ${ }^{3}$
}

${ }^{1}$ Department of Anthropology, Institute of Archaeology, Baylor University, Waco, TX, United States, ${ }^{2}$ Department of History, Honors College, Institute of Archaeology, Baylor University, Waco, TX. United States, ${ }^{3}$ Department of Geosciences, Watershed Studies Institute, Murray State University, Murray, KY, United States

Keywords: Critical Zone, complex systems, archeology, anthropocene, theory

\section{INTRODUCTION}

Recent conceptual advances in the Earth sciences have led to an improved understanding of the dynamics governing the Critical Zone (CZ) - the interface where life meets rock and soil on land (Brantley et al., 2007; Nordt and Driese, 2013). Among the key insights is a renewed appreciation for the deeply intertwined and non-linear nature of the processes in play, where small changes in one or two variable values or in their interactions can have large and often non-intuitive consequences, including the emergence of complex phenomena (Brantley et al., 2017). This application of complex systems (CS) methods and theory to conceptualize the CZ, both in part and in whole, places a premium on valid model construction. The potential analytical consequences of inaccurately modeling variables, processes, or interactions here though is dramatic, especially in light of the non-linear nature and coupled dynamics of CS models. In this brief note, we offer our opinion to the CZ science community that CS modeling of Holocene CZ processes and records (i.e., the paleo-CZ [pCZ]; Beverly et al., 2017) may be further refined-and perhaps must be further refined-through increased integration of archeological data and theory.

\section{THE ANTHROPOCENE}

While the specifics of the term "Anthropocene" are debated, there is nevertheless broad consensus that human activities have increasingly altered the $\mathrm{CZ}$ through the Holocene, culminating in global-scale perturbations to the modern CZ (Hooke, 2000; Montgomery, 2007; Wilkinson and McElroy, 2007). This stands in contrast to the Pleistocene record, which does not clearly evince hominins modifying either pCZ model dynamics or its resulting physical manifestations. This disconnect in the record reflects a dramatic shift in hominin agency, one in which Holocene humans began to modify energy-flow within the $\mathrm{pCZ}$ at ever grander scales. At issue, what changed? We and many others suggest that once humans began to modify energy flow via intensive agriculture and deforestation, humans were transformed from "casual passengers" of the pCZ (Ferraro, 2012) to potentially aggressive drivers of change within it. With regards to human activities, complex systems, and especially the $\mathrm{pCZ}$, several critical questions remain. Specifically how, when, where, and why did human behavior change the output of CZ models? Which suite of human complex systems were either partially or principally involved (e.g., socioeconomic, sociopolitical, technological, demographic, subsistence, ideological)? And, of particular interest here: how do these "archeological complex systems" interface with and influence pCZ models (see also Brantley et al., 2007)? That is, what were the dynamics of these interacting and evolving complex systems? 
To answer these questions will require an interdisciplinary approach, one in which archeology plays an important role (Brantley et al., 2007). For the presumed CZ audience of this paper, there is no need to belabor the inclusion of $\mathrm{CZ}$ in this research program. However, we note that while decades of geoarchaeological research has advanced our understanding of human-environmental interactions, there has been little attempt by $\mathrm{CZ}$ scientists to integrate archeology into their work. Despite Brantley et al.'s (2007) call to action, a Google search using the terms Critical Zone and Archaeology returns zero published papers focused on this topic as of May 2018. We aim here to outline why we believe archeology should be a required element of CZ research, as well as which aspects of archeology we believe should provide the greatest scientific returns. We see this as an introductory step toward increased interdigitation of the fields, with the common goal of better understanding humanenvironmental interactions.

\section{WHY ARCHEOLOGY?}

The potential value of archeological contributions to $\mathrm{pCZ}$ studies is substantial. While some aspects of human-environment interactions can be modeled and tested using a combination of currently observable processes and historical records (Hoggarth et al., 2017), archeology provides the single best access to the preliterate and prehistoric records of human activities. Accessing this record via archeology will dramatically expand the number and range of contexts that can be investigated within the framework of $\mathrm{CZ}$, as well as affording opportunities for longitudinal analyses of human-environmental interplay through time. This has several major benefits. Elucidating past human activities within the context of $\mathrm{pCZ}$ will allow us to move beyond simple explanations that "humans are responsible for geologicallyobservable changes to the pCZ" (e.g., Hooke, 2000). Instead these approaches will allow scholars to further delve into the cultureand locality-specific aspect(s) of human behavior, ecology, economy, and belief systems that were responsible for specific changes via specific pathways and series of complex interactions with non-linear outcomes. This shift toward significantly more mechanistic (i.e., CS) explanations that incorporate specific economic, cultural, and ideological factors holds benefit for both studies of the pCZ and Holocene archeology. In addition, when humanity's possible role in modifying the pCZ is equivocal, archeology can and should provide an invaluable additional source of potential information and insights into past human activities. In some instances, this may help resolve otherwise intractable equifinalities (Turner and Sabloff, 2012). It is salient too when dealing with the interplay of highly complex systems (human and CZ), wherein not all observable changes in the pCZ need be caused by extraordinarily visible earth science processes. For instance, it has recently been suggested that Middle Holocene denudation of northern Africa may have been accelerated following the intensification of pastoralism in the region (Wright, 2017). This is an intriguing hypothesis that refers to minimally visible activity (archeologically-speaking, at least) which nevertheless may have helped to facilitate quite dramatic changes to the pCZ. Further testing this and other such models will require the involvement of archeologists, CZ modelers, and traditional earth scientists working in concert.

\section{WHICH ARCHEOLOGY?}

So which aspects of human behavior might best help inform pCZ studies? We answer in three parts. At the most conceptuallevel, we suggest focusing on human behaviors that are most likely to redirect energy flow within the $\mathrm{CZ}$. The rationale here is clear: significant anthropogenic effects on energy flow within the CZ (a CS model driven by energy flow) should return complex outcomes of some sort. At issue here is determining exactly what that "sort" is, and how it is derived. At a proximatelevel, we suggest that human activities capable of affecting such change will likely accord with the interrelated triad of subsistence, demography, and technology. We note that these activities are themselves intertwined within "human" complex systems, where slight advances in one soon translate into advances in the others (Kohler, 2012). Slightly modifying one or more aspects of this triad and/or the CS model(s) within which they are embedded is then predicted to return complex, non-linear pCZ outcomes, the manifestations of which will vary by setting.

At even higher orders of investigation, an "ultimate-level" for some social scientists, it is interesting to relay that this triad itself will have both reflected and conditioned past sociopolitical and economic complexity, ideology, as well as a host of other factors. For example, imagine two large islands, identical in every respect, each inhabited by its own isolated culture group. One island has $10^{3}$ people, the other has $10^{6}$. With such differences in population sizes, we can safely assume radically different subsistence activities (with hunting and gathering on the former vs. agriculture on the latter), technology (with the latter possessing metallurgy, prestige goods, physical infrastructure development, and monumentality), as well as socio-political and economic organization (band-level vs. complex chiefdom or state) and ideology (egalitarian and animistic vs. stratified sociopolitical and economic inequality and organized religion), all of which scale predictably with population size. It is equally easy to posit how such differences in human activities and adaptations likely affected their respective pCZs. For example, one might predict evidence of extensive deforestation and associated topsoil loss on the latter island, with the former perhaps still largely resembling its pre-inhabited state. But what exactly in the traditional CZ or pCZ model has changed-if anything-that resulted in these islands having two distinct $\mathrm{pCZ}$ records?

Clearly the answer is human activity. Using a biological analogy, we suggest that anthropogenic modifications to the conceptual form of the pCZ (i.e., tweaking variables and interactions of variable within the pCZ CS model) are likely recorded within the physical form of the $\mathrm{pCZ}$ as earth science phenomena (a phenotype of a sort), conditioned by specific anthropogenic activities (a genotype of a sort), which itself reflects specific social, economic, and/or ideological adaptations (i.e., various alleles in our metaphor). Although this is an oversimplification, thinking in such Mendelian terms 

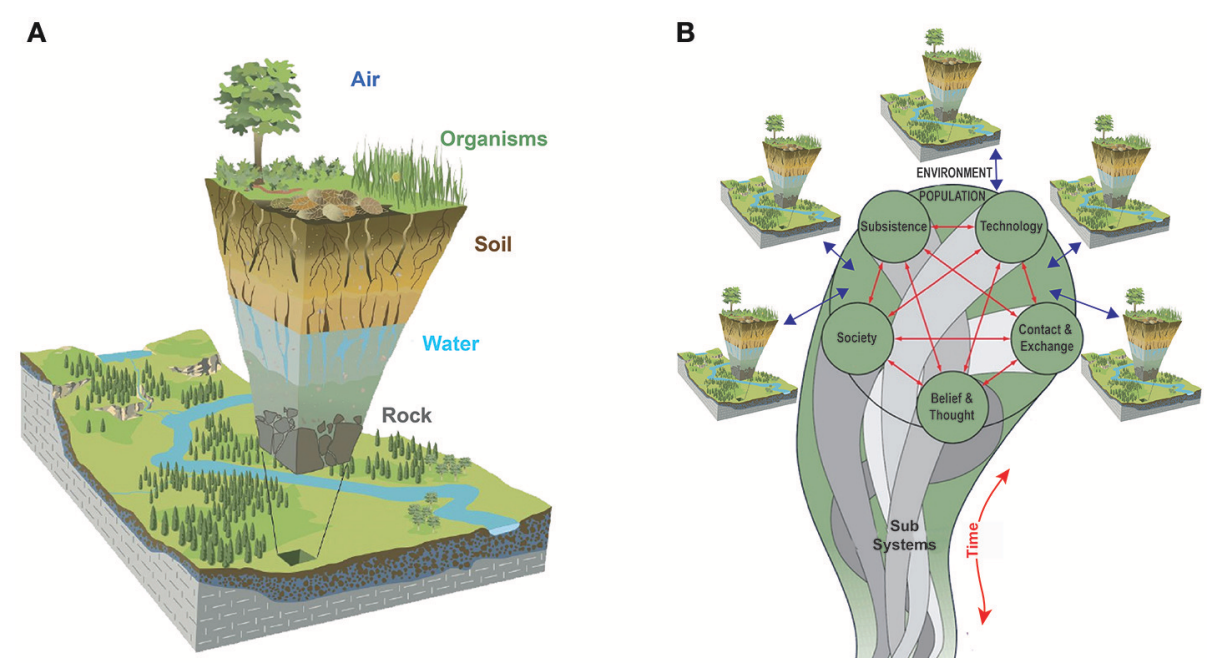

FIGURE 1 | Theoretical frames of reference. (A) An illustration showing the CZ and its components: air, organisms, soil, water, and rock (modified from Chorover et al. 2007), artwork by R. Kinlimann. (B) An illustration showing the interrelated sub-systems of human social systems, as well as the interactions between these systems and local hCZ's (modified from Renfrew and Bahn, 2016).

may accurately convey the nature of processes we envision. Nevertheless, human activities are undeniably components or variables (or at least passengers) of the pCZ CS, their effects potentially expressed under certain demographic-subsistencetechnological-and pCZ contexts (Figure 1). This has several implications.

(1) Studies of the material evidence of past human activities (i.e., archeological investigations) are ultimately indivisible from Holocene pCZ studies, and vice versa. This must be true if humans and their activities are active components of the pCZ CS, capable of modifying it. As such, we call for increased integration of the two disciplines, including explicitly incorporating human activities into all pCZ models, even if to simply falsify "anthropogenic influences" in a given case. Doing so will serve to scientifically delimit boundary conditions on past human-pCZ interactions, as well as allow us to rigorously explore the dynamics of such interactions when possible.

(2) For pCZ scholars, it strongly suggests that that the Holocene $\mathrm{pCZ}$ formed under different operating conditions (i.e., different $\mathrm{CZ}$ model conditions) than were present and active before the Holocene; the difference, of course, being anthropogenic impact. Given the CS model nature of the $\mathrm{CZ}$, where minor changes to a single variable and/or its interactions can have non-linear, non-intuitive, potentially complex outcomes, most or all parts of the "standard CZ model" could theoretically be influenced or modified by the emergence of impactful human populations. The nature and extent to which humans can modify this system is obviously of interest to both archeologists and earth scientists, and will require further investigation. To this end, we suggest that the $\mathrm{CZ}$ model for the Holocene (hCZ) should broaden "anthropogenic forcing" components of the model to specifically articulate (and then explore) the specific roles and interplay of human demography, subsistence, and technology, as well as sociopolitical, economic, and ideological adaptations within the $\mathrm{CZ}$ model context (Figure 1). Interestingly, this all leads to a specific prediction as to the timing and tempo, though not nature, of the effects of anthropogenic change to the hCZ: anthropogenic forcing should become increasingly powerful through time, closely mirroring human demographic changes and its correlates. This suggests that pCZ models without significant anthropogenic components will become increasingly inaccurate as their studies approach the present day, and ever increasingly so as they project into the future. This last point is particularly concerning, and highlights the pressing need to better understand the nature and dynamics of human-CZ interactions.

(3) For Holocene archeologists, it highlights the critically important role that they must play in studies of these human-CZ dynamics, as well as potentially provides an entirely new source of data for their investigations. For the former, archeology will often be the single best source of specific information on past human activities. For some activities, archeology may be the sole source of information. Moreover, as the hCZ is, in part, a product of human activities (Figure 1B), it should both reflect and record details of human social, economic, and ideological thoughts and processes (e.g., Kirch, 2007, 2011). This potentially provides a hitherto untapped source of information on the human past: one that we feel archeologists and CZ scientists should collaboratively explore further.

\section{CONCLUSIONS}

Archeologists and $\mathrm{CZ}$ scientists share a common goal to understand the world and our place within it. At issues here is how to best accomplish this goal. We contend that increased integration of these disciplines will yield a more comprehensive understanding of the totality of patterns and 
processes involved in the dynamics of the $\mathrm{CZ}$, the human past, and the interdigitation of the two. We hope that this paper may serve to stimulate valuable discussions and increased collaborative ventures amongst these scholars.

\section{REFERENCES}

Beverly, E. J., Peppe, D. J., Driese, S. G., Blegen, N., Faith, J. T., Tryon, C. A., et al. (2017). Reconstruction of late pleistocene paleoenvironments using bulk geochemistry of paleosols from the lake victoria region. Front. Earth Sci. 5:93. doi: 10.3389/feart.2017.00093

Brantley, S. L., Goldhaber, M. B., and Ragnarsdottir, K. V. (2007). Crossing disciplines and scales to understand the Critical Zone. Elements 3, 307-314. doi: 10.2113/gselements.3.5.307

Brantley, S. L., Lebedeva, M., Balashov, V., Singha, K., Sullivan, P. L., and Stinchcomb, G. (2017). Relating chemical reaction fronts to hillslope drainage. Geomorphology 277, 100-117. doi: 10.1016/j.geomorph.2016.09.027

Chorover, J., Kretzschmar, R., Garcia-Pichel, F., and Sparks, D. L. (2007). Soil biogeochemical processes within the Critical Zone. Elements 3, 321-326. doi: 10.2113/gselements.3.5.321

Ferraro, J. V. (2012). A primer on Paleolithic technology. Nat. Educ. Knowl. 4, 9.

Hoggarth, J. A., Restall, M., Wood, J. W., and Kennett, D. J. (2017). Drought and its demographic effects in the Maya Lowlands. Curr. Anthropol. 58, 82-113. doi: $10.1086 / 690046$

Hooke, R. L. (2000). On the history of humans as geomorphic agents. Geology 28, 843-846. doi: 10.1130/0091-7613(2000)28\&lt;843:OTHOHA\&gt;2.0.CO;2

Kirch, P. V. (2007). Hawaii as a model system for human ecodynamics. Am. Anthropol. 109, 8-26. doi: 10.1525/aa.2007.109.1.8

Kirch, P. V. (2011). Roots of Conflict: Soils, Agriculture, and Sociopolitical Complexity in Ancient Hawai i. School for Advanced Research Advanced Seminar Series. Santa Fe, NM: School for Advanced Research Press.

Kohler, T. A. (2012). Complex systems and archaeology. Archaeol. Theor. Today 93-123.

\section{AUTHOR CONTRIBUTIONS}

JF, JH, DZ, KB, and GS jointly conceived, wrote, and edited the manuscript and produced the figure.

Montgomery, D. R. (2007). Soil erosion and agricultural sustainability. Proc. Natl. Acad. Sci. U.S.A. 104, 13268-13272. doi: 10.1073/pnas.0611508104

Nordt, L. C., and Driese, S. G. (2013). Application of the Critical Zone concept to the deep-time sedimentary record. Sediment. Record 11, 4-9. doi: $10.2110 /$ sedred.2013.3

Renfrew, C., and Bahn, P. (2016). Archaeology: Theories, Methods, and Practice. London: Thames and Hudson.

Turner, B. L., and Sabloff, J. A. (2012). Classic Period collapse of the central Maya Lowlands: insights about human-environment relationships for sustainability. Proc. Natl. Acad. Sci. U.S.A. 109, 13908-13914. doi: 10.1073/pnas.12101 06109

Wilkinson, B. H., and McElroy, B. J. (2007). The impact of humans on continental erosion and sedimentation. GSA Bull. 119, 140-156. doi: 10.1130/B 25899.1

Wright, D. K. (2017). Humans as agents in the termination of the African humid period. Front. Earth Sci. 5:4. doi: 10.3389/feart.2017.00004

Conflict of Interest Statement: The authors declare that the research was conducted in the absence of any commercial or financial relationships that could be construed as a potential conflict of interest.

Copyright (C) 2018 Ferraro, Hoggarth, Zori, Binetti and Stinchcomb. This is an openaccess article distributed under the terms of the Creative Commons Attribution License (CC BY). The use, distribution or reproduction in other forums is permitted, provided the original author(s) and the copyright owner(s) are credited and that the original publication in this journal is cited, in accordance with accepted academic practice. No use, distribution or reproduction is permitted which does not comply with these terms. 